\title{
ION PRODUCTION AND TUNE SHIFT IN THE RECYCLER RING
}

\author{
K. Gounder *, J. Marriner, and S. Mishra \\ FNAL, Batavia, IL 60510, USA.
}

\section{Abstract}

We calculate the ion production rate for a beam of 2 $\times 10^{12}$ antiprotons in the Recycler Ring using the known vacuum residual gas composition. We study the effect of ion buildup around the antiproton beam on beam lifetime and stability. We compare the ion production rate with the actual measurement using the beam tune shifts when the RF gap in the circulating beam is removed. Work supported by the U.S. Department of Energy under contract No. DE-AC02-76CH03000.

\section{INTRODUCTION}

The Recycler Ring [1] located in the upper portion of the Main Injector tunnel at Fermilab is designed as a storage ring for antiprotons. Antiprotons transferred from the Accumulator and the residual Tevatron stores will be cooled and stored in the Recycler Ring before reinjection into the Tevatron. The stacking rate of the antiprotons in the Accumulator is improved by steady transfer of antiprotons to the Recycler Ring when the stack size becomes sufficiently large. Thus, as a part of Run II upgrade, the Recycler Ring is expected to provide a factor of 2-3 improvement in luminosity. Pressently, the Recycler Ring (RR) is being commissioned using protons as well as antiprotons. As a beginning phase of the integration of RR in the Fermilab collider complex, we expect to have a circulating beam of $2 \times 10^{12}$ antiprotons. From the known residual gas composition of the RR vacuum, we study the ion production rates and the effects of ion trapping by the antiproton beam on lifetime and transverse tunes. The relevant RR parameters used are listed in Table 1. More detailed description of the Recycler Ring can be found elsewhere [1].

\begin{tabular}{|l|c|}
\hline Paramater & Value \\
\hline Acceptance (mm-mr) & $40.0 \pi$ \\
Average $\beta(\mathrm{m})$ & 40.0 \\
Average beam pipe radius (in $\mathrm{m})$ & 0.023 \\
Beam energy $(\mathrm{GeV})$ & 8.89 \\
Average beam $\beta$ & 0.998 \\
Average beam $\gamma$ & 9.48 \\
Maximum energy loss $(\mathrm{GeV})$ & 0.089 \\
\hline
\end{tabular}

Table 1: The basic Recycler Ring parameters relevant for the computations detailed in this note.

* gounder@fnal.gov

\section{RECYCLER RING VACUUM RESIDUAL GASES}

The RR ultra high vacuum is maintained by arrays of Titanium sublimation pumps (TSP) and Ion pumps has a total pressure of a fraction of a nano Torr. The types of gases present in the vacuum and their partial pressures can be obtained from RGA measurements and Ion gauge readings done often around the ring. The major constitutents of the vacuum (average of RGA measurements normalized by Ion gauge readings) are listed below. We have also detected some very minor quantities of hydro carbons such as Ethane, Etheylene etc. and denoted them as the 'unknown' component. The observed gases and the relevant parameters are listed in Table 2.

\begin{tabular}{|l|c|c|}
\hline Gas & $\begin{array}{c}\text { Avg. Pres. } \\
\text { [Torr] }\end{array}$ & $\begin{array}{c}\text { Gas Density } \\
{\left[M^{-3}\right]}\end{array}$ \\
\hline $\mathrm{H}_{2}$ & $0.354 \mathrm{E}-09$ & $1.25 \mathrm{E}+13$ \\
$\mathrm{H}_{2} \mathrm{O}$ & $0.380 \mathrm{E}-10$ & $1.35 \mathrm{E}+12$ \\
$\mathrm{CO}$ & $0.190 \mathrm{E}-10$ & $6.73 \mathrm{E}+11$ \\
$\mathrm{Ar}$ & $0.010 \mathrm{E}-10$ & $3.54 \mathrm{E}+10$ \\
$\mathrm{CH}_{4}$ & $0.050 \mathrm{E}-10$ & $1.77 \mathrm{E}+11$ \\
$\mathrm{CO}_{2}$ & $0.150 \mathrm{E}-10$ & $5.31 \mathrm{E}+11$ \\
Unknown & $0.105 \mathrm{E}-09$ & $3.72 \mathrm{E}+12$ \\
\hline Total & $0.537 \mathrm{E}-09$ & $1.90 \mathrm{E}+13$ \\
\hline
\end{tabular}

Table 2: The Recycler Ring vacuum residual gases as measured by RGA and Ion gauges located around the ring. The 'unknown' component will be treated as Nitrogen for computational purposes.

\section{ION PRODUCTION RATES}

The rate of ionization $R_{j}$ for a given gas type can be written as [3]:

$$
R_{j}=n_{j} n_{b} \sigma_{j} v
$$

with $n_{j}$ density of the gas type, $n_{b}$ beam particle density, $\sigma_{j}$ ionization cross section and $v$ the beam particle velocity. The $\sigma_{j}$ can be parametrized:

$$
\sigma_{j}=4 \pi\left(\frac{\hbar}{m_{e} c}\right)^{2}\left\{M_{j}^{2}\left[\frac{1}{\beta^{2}} \ln \left(\frac{\beta^{2}}{1-\beta^{2}}\right)-1\right]+\frac{C_{j}}{\beta^{2}}\right\}
$$

where $\hbar$ is Planck's constant, $m_{e}$ the electron mass, c the speed of light and $\beta$ associated with the ionizing beam. The parameters $M_{j}^{2}$ and $C_{j}$ have been tabulated for various types of gases [4]. Knowing the partial pressure of 
each gas as given Table 2 and beam distribution for a beam of $2 \times 10^{12}$ antiprotons, the total ion production rate can be computed. The rate for the above composition of gases considered is $5.0 \times 10^{8} /$ meter $/$ second. Now for the whole ring, this becomes $1.7 \times 10^{12}$ ions/second - or each beam particle roughly creates an ion per second in the ring.

\section{THE TUNE SHIFT DUE TO IONS}

The presence of ion cloud around the beam can induce an incoherent tune spread due to the static transverse electrostatic field generated by the cloud. For a gaussian beam of longitudinal density $\lambda_{b}$, the transverse beam density $n_{b}(r)$ is given by:

$$
n_{b}(r)=\frac{-\lambda_{b}}{2 \pi \sigma_{r}^{2}} e^{-\frac{r^{2}}{2 \sigma_{r}^{2}}}
$$

where $\sigma_{r}$ is the radial width of the distribution. Assuming similar distribution for the ion cloud, the electric field (beam fully neutralized) generated by the ions is given by:

$$
E_{r}(r)=\frac{-q^{2} \lambda_{b}}{2 \pi \epsilon_{0} r}\left\{1-e^{-\frac{r^{2}}{2 \sigma_{r}^{2}}}\right\}
$$

where $\epsilon_{0}$ is the permittvity for free space and q, unit charge. The radial space charge force $F_{r}(r)$ for small distances can be by obtained using Lorentz equation as:

$$
F_{r}(r)=\frac{q^{2} \lambda_{b}}{4 \pi \epsilon_{0} \sigma_{r}^{2}} r
$$

Converting this result into the form of a quadrupole gradient error $\Delta K$ as:

$$
\Delta K=\frac{r_{0} \lambda}{\gamma \beta^{2} \sigma^{2}}
$$

The tune shift due to this gradient error can be cast as:

$$
\Delta \nu=\frac{1}{4 \pi} \int d s \beta(s) \Delta K
$$

where $r_{0}$ is the classical proton radius. For simplicity, we assume an average beta of 40 meters around the ring, and for a beam of $1.0 \times 10^{12}$ antiprotons with total ionization case, the transverse tune shift will be:

$$
\Delta \nu=\frac{N \beta_{a v g} r_{0}}{\gamma \beta^{2} 4 \pi \sigma^{2}} \approx 0.021
$$

\section{MEASUREMENT RESULTS}

Measurements were carried out with a beam of $\approx 2 \times 10^{11}$ antiprotons in the Recycler Ring. The longitudinal gap usually left in the beam is 64 RF buckets. The transverse tunes and beam lifetime were measured using Schottky and beam current monitors. After turning off the gap, lifetime and transverse tunes were remeasured. Then the tune Trambone was used to reset the tunes to its original values. The transverse tune and lifetime measurements were repeated. The measurement results are summarized in Table 3.

\begin{tabular}{|l|c|c|c|}
\hline \multicolumn{1}{|c|}{ Scenario } & $\begin{array}{c}\tau_{a p} \\
\text { [hours] }\end{array}$ & $\begin{array}{c}\text { Transverse Tunes } \\
\text { [Fractional] }\end{array}$ & Beam \\
\hline \hline $\begin{array}{l}\text { Longitudinal } \\
\text { beam gap on }\end{array}$ & 135 & $(0.4166,0.4193)$ & $2.00 \mathrm{E}+11$ \\
\hline $\begin{array}{l}\text { No longitudinal } \\
\text { beam gap }\end{array}$ & 86 & $(0.4180,0.4215)$ & $1.91 \mathrm{E}+11$ \\
\hline $\begin{array}{l}\text { No longitudinal } \\
\text { beam gap but } \\
\text { tunes reset }\end{array}$ & 120 & $(0.4166,0.4193)$ & $1.90 \mathrm{E}+11$ \\
\hline
\end{tabular}

Table 3: Antiproton lifetime $\left(\tau_{a p}\right)$ and transverse tunes (horizontal, vertical) measurements with and with out the longitudinal gap in the circulating beam of $\approx 2.0 \times 10^{11}$ antiprotons in the Recycler Ring. The measurements indicate tune shifts due to ion trapping.

\section{THE EFFECT OF IONS ON BEAM LIFETIME}

To study the additional beam-gas scattering due to ion trapping, let us assume that about $2.0 \times 10^{12}$ antiprotons circulating in RR with a gaussian beam shape of $\sigma_{r}=$ $0.005 \mathrm{~m}$. Now assuming the ionization rate is complete that each antiproton traps an ion, there will be $2.0 \times 10^{12}$ ions trapped along the beam with a similar gaussian distribution. To estimate the maximum effect, the ions are taken to have a similar composition as the normal vacuum shown in Table 2. Using the convention that all the beam resides in the $\pm 3 \sigma_{r}$ region, we expect all the ions to be contained in a tube of radius $3 \sigma_{r}$ along the ring (3320 meters in length).Therefore the average ion density for each gas can be written as in Table 4 .

\begin{tabular}{|l|c|c|c|}
\hline Ions & Gas Fraction & $\begin{array}{c}\text { Ion Density } \\
{\left[M^{-3}\right]}\end{array}$ & $\begin{array}{c}\text { Par. Pres. } \\
\text { [Torr] }\end{array}$ \\
\hline $\mathrm{H}_{2}$ & 0.66 & $5.62 \mathrm{E}+11$ & $1.59 \mathrm{E}-11$ \\
$\mathrm{H}_{2} \mathrm{O}$ & 0.07 & $0.60 \mathrm{E}+11$ & $1.69 \mathrm{E}-12$ \\
$\mathrm{CO}$ & 0.04 & $0.34 \mathrm{E}+11$ & $0.96 \mathrm{E}-12$ \\
$\mathrm{Ar}$ & 0.00 & $0.00 \mathrm{E}+11$ & $0.00 \mathrm{E}-12$ \\
$\mathrm{CH}_{4}$ & 0.01 & $0.09 \mathrm{E}+11$ & $0.03 \mathrm{E}-12$ \\
$\mathrm{CO}_{2}$ & 0.03 & $0.26 \mathrm{E}+11$ & $0.07 \mathrm{E}-12$ \\
Unknwon & 0.19 & $1.61 \mathrm{E}+11$ & $0.45 \mathrm{E}-11$ \\
\hline Total & 1.00 & $8.52 \mathrm{E}+11$ & $2.31 \mathrm{E}-11$ \\
\hline
\end{tabular}

Table 4: Average Ion densities trapped around the beam assuming the extreme case of full neutralization of a beam of $2.0 \times 10^{12}$ antiprotons. The ion distribution is assumed to be same as that of the beam - Gaussian with a $\sigma_{r}=0.005$ meters. The vacuum gas composition given in Table 2 is used here.

Comparing two tables above we see that the partial pressures are roughly two orders of magnitude smaller for ions compared to that of normal gases. At this level we can 
assume that this additional density the particles see along with normal gas densities. On the otherhand, if we assume that we can stack an order of magnitude higher, then we may not be able to treat the ion densities as independent of the normal gas densities! But in this case, we assume that they are independent, and compute the lifetime to compare with the case of no ion trapping.

The lifetime due to beam-gas scattering has four components: (a) Single coloumb scattering where a particle is lost due to a single scattering of beam particle with a gas atom; (b) Multiple coloumb scattering when particle is lost at the beam pipe limiting aperture due to emittance growth of the beam atttributed to multiple scattering of beam with gas atoms ; (c) Nuclear scattering when a beam particle is absorbed by the nucleus of a gas atom; and (d) Inelastic scattering when a particle is lost due to energy loss from gas atom ionization, bremsstrahlung etc. All four types of processes of beam loss can be combined to produce the total lifetime of the beam. The lifetime due to multiple scattering process is computed for the asymptotic case when the beam is already grown and filled the whole beam pipe aperture. More details of the lifetime computations can be found elsewhere [5]. We use RR parameters listed in Table 1 along with gas densities shown in Tables 2 and 4. The results of lifetime estimations are summarized in Table 5.

\begin{tabular}{|l|c|c|}
\hline Physical Process & $\begin{array}{c}\text { No Ions } \\
\text { (Normal Case) } \\
\text { [hours] }\end{array}$ & $\begin{array}{c}\text { Neutralization } \\
(100 \%) \\
\text { [hours] }\end{array}$ \\
\hline \hline Single Coloumb & $4.64 \times 10^{2}$ & $4.62 \times 10^{2}$ \\
\hline Inelastic Scatt. & $8.06 \times 10^{2}$ & $8.02 \times 10^{2}$ \\
\hline Mult. Coloumb & $5.55 \times 10^{1}$ & $5.53 \times 10^{1}$ \\
\hline Nuclear Scatt. & $1.61 \times 10^{3}$ & $1.60 \times 10^{2}$ \\
\hline Total Life Time & $4.54 \times 10^{1}$ & $4.50 \times 10^{1}$ \\
\hline
\end{tabular}

Table 5: The lifetime comparisons with full neutralization of the $2.0 \times 10^{12}$ antiproton beam with case of no neutralization. The vacuum residual gas composition in Table and ion densities shown in Table 4 were used for the above lifetime estimations.

From Table 5, the effect of full neutralization is small on lifetime due to beam-gas scattering for a beam of $2.0 \times 10^{12}$ antiprotons, less than $1 \%$ ! For a beam of $2.0 \times 10^{13}$ antiprotons, this could become easily noticeable - about $8 \%$ !

\section{ION CLEARING IN THE RECYCLER RING}

If the ions produced are not effectively cleared from beam pipe, this will affect the beam stability by tune shifts, induce coherent ion-beam transverse oscillations etc. The ion cloud will increase the concentration of gas molecules near the beam and can cause additional beam-gas scattering affecting beam lifetime adversely. This effect as shown above may be considerable for higher intensity low emittance beams. The trapped ions are cleared in the RR using two sets of ion clearing electrodes located in each half cell. In addition to the clearing electrodes, a longitudinal gap in the circulating antiproton beam is maintained using RF cavities. Also transverse dampers will be installed in the RR to combat coherent instabilities due to beam-ion oscillations. They are designed to have electronics capable of exciting the beam at the resonant frequency of the ion oscillations about the beam centroid.

\section{REFERENCES}

[1] Gerry Jackson, "The Fermilab Recycler Ring Technical Design Report", November 1996, Fermilab-TM-1991; Mishra, "Status of Fermilab Recycler Ring", EPAC2002, Paris, June 2002.

[2] J. Marriner et. al., "Run II Handbook", at http://wwwbd.fnal.gov/runII/index.html; Also see K. Gounder, "The Status of Run II at Fermilab", in the 2002 proceedings of ICHEP, Amsterdam, July 2002, Fermilab-Conf-02-233-E.

[3] A. Poncet in the Proceedings of CERN Accelerator School, Vol. 2, 859-878, CERN-95-06.

[4] Rudd et al., Rev. Mod. Phys., Vol. 57, No.4, 1985; F Rieke and W. Prepejchal, Phys. Rev. A, 6, 1507 (1972).

[5] K. Gounder et al., "Recycler Ring Beam Lifetime", FermilabConf-01-186-E, PAC-2001-RPPH055, July 2001. 